Yang Zhang

\title{
Über die Trinkkultur in chinesischen und deutschen Trinkgedichten - am Beispiel von Trinkgedichten Li Bais und Johann Wolfgang von Goethes
}

\begin{abstract}
Das Trinken als Nahrungsaufnahme ist ein kulturell-psychisch-gesellschaftlich geprägter Akt und geht weit über seine Funktion für den Stoffwechsel hinaus. Um das Trinken herum ist die Trinkkultur entstanden, die im Kollektivgedächtnis einer Menschengruppe gespeichert wird und in ihre Kultur integriert ist. Im vorliegenden Beitrag handelt es sich um eine vergleichende Untersuchung der chinesischen und der deutschen Trinkkultur anhand einiger Trinkgedichte Li Bais und Johann Wolfgang von Goethes. Es lässt sich feststellen, dass die chinesische und die deutsche Trinkkultur trotz der bestehenden Ähnlichkeiten deutliche Unterschiede aufweisen.
\end{abstract}

Keywords: Trinkkultur, Trinkgedicht, Li Bai, Johann Wolfgang von Goethe

\section{Einleitung}

Das Trinken als Nahrungsaufnahme ist auch ein kulturell-psychisch-gesellschaftlich geprägter Akt und steht in engem Zusammenhang mit der Geschichte einer Kultur und mit der Entstehung und Prägung kollektiver Identitäten (vgl. Wierlacher 2003, 165-166). Was die Trinkkultur angeht, können sowohl China als auch Deutschland bzw. Europa auf eine sehr lange Geschichte zurückblicken. In der chinesischen wie in der deutschen Sprache haben jeweils die beiden Begriffe „Jiu酒“ und „Wein“ nicht wenige Spuren hinterlassen, z. B. das Sprichwort im chinesischen Volksmund 酒后吐真言 [wörtlich: ,Nach dem Trinken folgt die wahre Rede'] und seine Übersetzungsentsprechung im Deutschen „Im Wein liegt die Wahrheit“, die eigentlich von dem lateinischen Satz In vino veritas stammt. Die beiden Sprichwörter basieren auf der Lebenserfahrung, dass niemand effektiv lügen

\footnotetext{
Anmerkung: Die vorliegende Arbeit stellt Teilforschungsergebnisse vom Projekt Übertragung und Erforschung der Tang-Dichtung im deutschsprachigen Raum (Projekt-Nr. 17XZW025, gefördert vom NSSFC) dar. 本文系 2017 年度国家社科基金西部项目“唐诗在德语世界的译介与研究”（项 目编号: 17XZW025）的阶段性成果。

Ә Open Access. (C) 2020 Yang Zhang, publiziert von De Gruyter. (c) BY-NC-ND Dieses Werk ist lizenziert unter der Creative Commons Attribution-NonCommercial-NoDerivatives 4.0 Lizenz. https://doi.org/10.1515/9783110641998-021
} 
könnte, wenn er betrunken ist. Über die Entstehung der Trinkkultur gibt es in China und im Westen unterschiedliche Überlieferungen. Im ersten Zeichenlexikon der chinesischen Schrift Shuowen Jiezi (说文解字), das um das Jahr 100 n. Chr. von dem Gelehrten Xu Shen (许慎) der Östlichen Han-Dynastie zusammengestellt und im Jahr 121 veröffentlicht wurde, wird die Entstehung von Jiu酒 wie folgt erklärt:

古者儀狄作酒醪，禹嘗之而美，遂疏儀狄。杜康作秝酒。(Xu 2017, 1225)

[Wörtlich: ,Im Altertum stellte Yi Di ein alkoholhaltiges Getränk aus Klebreis her. Yu der Große ${ }^{1}$ probierte dieses Getränk und lobte den Geschmack, aber in der Folge entfremdete er sich Yi Di. ${ }^{2}$ Du Kang stellte den Schnaps aus Sorghum her.']

Nach dieser Erklärung werden in China allgemein Yi Di (儀狄) und Du Kang (杜康) als Erfinder des Alkoholbrennens betrachtet. In der westlichen Kultur wird die Entstehung von Wein oft mit Dionysos in Verbindung gebracht, einem Gott des Weines, der Freude, der Trauben, der Fruchtbarkeit usw. in der griechischen Götterwelt. Aus seiner großen Liebe zum Wein hat er die Menschen die Herstellung des Weines gelehrt. Im antiken Kulturraum wurden auch verschiedene Feste zu Ehren von Dionysos abgehalten, bei denen der Wein immer im Mittelpunkt stand. Da das Volksleben in China und im Westen bzw. in Deutschland vom Trinken stark geprägt ist, spiegelt sich die Trinkkultur auch sehr häufig in der Literatur wider. Li Bai und Johann Wolfgang von Goethe, jeweils die herausragenden Repräsentanten der chinesischen und der deutschen Literatur, waren beide bekanntlich dem Trinken sehr zugetan und schufen unter dessen Einfluss viele schöne Trinkgedichte, die bis heute immer noch als Kostbarkeiten der Weltliteratur gelten und landesweit, ja weltweit verbreitet sind. Vor diesem Hintergrund wird in der vorliegenden Arbeit der Versuch unternommen, die Trinkgedichte von Li Bai und Johann Wolfgang von Goethe einer komparativen Analyse zu unterziehen und aufgrund dessen die Trinkkultur von China und Deutschland (und ggf. Europa), die in den Gedichten ihren Niederschlag findet, aus interkultureller Perspektive $\mathrm{zu}$ interpretieren.

$1 \mathrm{Da} \mathrm{Yu}, \mathrm{Yu}$ der Große, war der Legende nach der erste Kaiser der mythischen Xia-Dynastie und ist der sechste der mythischen Urkaiser Chinas, die als Kulturheroen und Begründer der chinesischen Zivilisation gelten.

2 Weil ihm dieses Getränk so gut schmeckte, war Yu der Große der Meinung, dass es später bestimmt die Kaiser gebe, die dem Trinken erliegen und daher die Staatsangelegenheiten vernachlässigen könnten, und hielt dieses neue Produkt für nicht gut. 


\section{Die Dichter und das Trinken}

Wie oben schon erwähnt, hatten Li Bai und Johann Wolfgang von Goethe eine Vorliebe fürs Trinken. Li Bai, einer der bedeutendsten lyrischen Dichter Chinas in der Tang-Zeit, wird im Volksmund sowohl als „Shixian“ (诗仙, wörtlich: ,Unsterblicher der Dichtkunst‘), wie auch als „Jiuxian“ (酒仙, wörtlich: ,Unsterblicher des Weines') bezeichnet. Sein letzterer Beiname drückt die enge Beziehung zwischen dem Dichter und dem Trinken schon deutlich aus. In der Bewunderung für Li Bais Schaffenskraft beim Trinken schrieb Du Fu, ein ähnlich bedeutender Dichter der Tang-Zeit, in seinem Gedicht Das Lied von den Acht Unsterblichen beim Zechen (饮中八仙歌) Folgendes:

$\begin{array}{ll}\text { 李白一斗诗百篇, } & \begin{array}{l}\text { Li Bo mit einer Kufe Wein, } \\ \text { das gibt dreihundert Gedichte. }\end{array} \\ \text { 长安市上酒家眠, } & \begin{array}{l}\text { In Tschang-an auf dem Marktplatz liegt } \\ \text { er schlafend in den Schenken. }\end{array} \\ \text { 天子呼来不上船, } & \begin{array}{l}\text { Als ihn der Himmelssohn einst rief, } \\ \text { erklomm das Boot er nicht. }\end{array} \\ \text { 自称臣是酒中仙。 } & \begin{array}{l}\text { Dein Untertan, so sprach er, ist } \\ \text { Ein Genius, tief im Wein! }\end{array}\end{array}$

Im Vergleich zu Li Bai hat Johann Wolfgang von Goethe, einer der bedeutendsten Repräsentanten deutschsprachiger Dichtung und des deutschen Geistes überhaupt, schon zu Anfang seines Lebens mit dem Wein Bekanntschaft gemacht. Seiner Geburtsanekdote zufolge war Goethe am 28. August 1749 bei seinem Eintritt ins Leben wegen der Ungeschicklichkeit der Hebamme fast tot und ohne Lebenszeichen. Aber nachdem man ihn in einen sogenannten Fleischarden mit Wein gelegt und ihm die Herzgrube mit Wein gebläht hatte, schlug er die Augen auf (vgl. Christoffel 1978, 10). Auch in der Kindheit und Jugend kam Goethe immer wieder mit Wein in Kontakt. Frankfurt am Main, Goethes Geburtsort, war schon damals eine Stadt des Weinhandels. Auch die Familie Goethe hatte viel mit Wein und Weinhandel zu tun. Aufgewachsen in einer solchen Familie, erwarb Goethe nicht nur gute Kenntnisse über den ganzen Prozess von der Traubenlese bis hin zur Abfüllung und Pflege - und der Qualität - der Weine, sondern er wuchs auch mit Sympathie für den Wein auf.

3 Das chinesische Original stammt von Du Fu 1983, 7. Übersetzt von Günter Debon (vgl. Debon 2009, 6). 
Über die Tagesmenge der beiden tüchtigen Trinker gibt es immer noch keine allgemein anerkannten Angaben, aber man kann sowohl in Gedichten wie auch in den Worten anderer wohl ein paar Hinweise darauf finden. In seinem Gedicht Das Lied von Hsiang-yang (襄阳歌) schrieb Li Bai:

\begin{tabular}{|c|c|}
\hline 鸬兹杓, 鹏鹉杯。 & $\begin{array}{l}\text { Mit dem Reiher-Schöpfer - } \\
\text { Mit dem Papageien-Becher - }\end{array}$ \\
\hline 百年三万六千日, & In den 36000 Tagen von hundert Jahren, \\
\hline 一日须倾三百杯。 & $\begin{array}{l}\text { Muss man an jedem Tag dreihundert Becher } \\
\text { leeren. }\end{array}$ \\
\hline 看汉水鸭头绿, & $\begin{array}{l}\text { Wenn man das entenkopfgrüne Wasser des } \\
\text { Hanflusses aus der Ferne betrachtet, }\end{array}$ \\
\hline 初酦醅。 & $\begin{array}{l}\text { So ist es so grün wie gerade gekelterter } \\
\text { Traubenwein. }{ }^{4}\end{array}$ \\
\hline
\end{tabular}

In diesem Gedicht war das lyrische „Ich“ schon stockbetrunken. In seiner Trunkenheit dachte es sogar darüber nach, dass man im Leben von hundert Jahren täglich viel trinken sollte. Die Zahl „dreihundert“ in dieser Zeile spielt auf den großen Gelehrten Zheng Xuan (郑玄) der Östlichen Han-Dynastie (25-220) an, der angeblich ein sehr tüchtiger Trinker war und einmal nacheinander dreihundert Becher geleert haben soll ${ }^{5}$, aber meines Erachtens dient sie hier eher zur Betonung von „viel“. Es gibt in der chinesischen Dichtkunst (allemal seit Tao Qian (陶潜)) eine ehrwürdige Tradition des Dichters als Trinker, sich angetrunken oder gar betrunken zu geben, also einen poetischen Gestus darzustellen, mit dem sich das lyrische Ich eine gewisse Aura der Unkonventionalität und Unkontrolliertheit gibt. So dürfen die „dreihundert Becher“ auf keinen Fall als die Tagesmenge von Li Bais Jiu-Konsum interpretiert werden.

Goethe selber hat sich nicht über seine genaue Tagesmenge geäußert; das haben andere getan, wie z.B. der englische Goethebiograph Lewes: „Den Wein liebte er sehr, täglich trank er seine zwei bis drei Flaschen“ (zit. nach Christoffel 1978, 18). Nach einer anderen Quelle trank er regelmäßig um 10 Uhr ein Glas Süßwein, dann im weiteren Verlauf des Vormittags ein Wasserglas guten Weines, mittags leerte er allein eine Flasche und zum Dessert manchmal noch ein Glas Champagner (vgl. Boehncke und Seng 2014, 79).

4 Das chinesische Original stammt von Li Bai 1983, 20. Übersetzt von Erwin Ritter von Zach (vgl. Von Zach 2007, 175).

5 Vgl. Li Bai 1983, 20. 
Überdies muss darauf hingewiesen werden, dass „,iu酒“ und „Wein“ keine 1:1-Entsprechung darstellen. Das chinesische Wort „Jiu酒“ bezieht sich auf alle alkoholhaltigen Getränke inklusive Wein. Eigentlich kann die Traubenweinkultur auch in China auf eine lange Geschichte zurückblicken; erstmals wurde in der Westlichen Han-Dynastie (202 v. Chr.-8. n. Chr.) an der westlichen Peripherie Chinas Traubenwein produziert, nachdem Zhang Qian (张寒), der chinesische Entdecker und kaiserliche Gesandte zur Zeit der Westlichen Han-Dynastie, Exemplare der Kulturrebe Vitis vinifera von einer seiner ausgiebigen, mehrjährigen Expeditionen aus Dayuan, westlich des Tarim-Beckens, nach China zurückgebracht haben soll (vgl. Kupfer 2011, 380; Wang und Huang 2005, 138-139). Die Traubenweinproduktion verbreitete sich allmählich über das ganze chinesische Kaiserreich und erreichte während der Tang-Dynastie (618-907) den Höhenpunkt. Auch Li Bai, jener Unsterbliche der Dichtkunst, soll eine große Vorliebe für den Traubenwein gehabt haben, wie sie in einigen seiner Trinklieder als Motiv zum Ausdruck gekommen ist, so in dem oben zitierten Gedicht Das Lied von Hsiang-yang. In den nachfolgenden Epochen der Song-, Yuan- und Ming-Dynastie (vom zehnten bis zum siebzehnten Jahrhundert) spielte der Traubenwein im Alltag, in der Literatur und Kunst zwar noch eine gewisse Rolle, erfuhr sogar noch einen Aufschwung während der Yuan-Zeit (1279-1368), verlor jedoch neben den anderen Varianten alkoholischer Getränke seine Bedeutung und geriet schließlich in Vergessenheit, bis es im Kontext der Globalisierung zu einer Renaissance der Weinkultur in China kam, freilich mit einem bis heute noch dominierenden Image als „westlichem Kulturgut“ (Kupfer 2011, 381). Die gängigste Alkoholsorte im heutigen China ist Baijiu (白酒, wörtlich: ,weißer Alkohol', soviel wie Schnaps), der vorwiegend aus Weizen oder Sorghum gebrannt wird. Was Li Bai damals trank, war selten Wein aus Trauben oder der im heutigen China populäre Schnaps. Das damals populärste Alkoholgetränk wurde aus gegorenem Korn hergestellt und verfügte über einen ähnlichen Geschmack wie der heutige Reiswein mit einem Alkoholgehalt von 5 Volumenprozent bis über 20 Volumenprozent, was deutlich unter dem Alkoholgehalt des heutigen Schnaps in China liegt (vgl. Guo 2012, 57). Es lässt sich feststellen, dass Jiu酒 in China meistens aus Getreide hergestellt wird, während man in Deutschland den Wein aus Trauben gewinnt.

Li Bais Vorliebe fürs Trinken von Jiu酒 und Goethes Freude am Wein lassen sich wohl auch dadurch begründen, dass sie die anregende Wirkung des Jiu oder des Weines auf das geistige Schaffen am besten kannten und am höchsten schätzten. Dies hat Goethe während seines Gesprächs am 11. März 1828 mit Johann Peter Eckermann explizit zum Ausdruck gebracht: „Es liegen im Wein allerdings produktivmachende Kräfte sehr bedeutender Art; aber es kommt dabei alles auf Zustände und Stunde an, und was dem einen nützt, schadet dem anderen." (Christoffel 1978, 22) 
Offensichtlich gehört Goethe selber zu „dem einen“. In seiner Autobiographie Aus meinem Leben. Dichtung und Wahrheit hat Goethe sich an die Entstehung einer seiner Jugenddichtungen erinnert, dass er „bei einer Flasche guten Burgunders, das ganze Stück wie es jetzt daliegt, in einer Sitzung niederschrieb“ (Von Goethe 2007b, 583). Genau wie Goethe, profitierte auch Li Bai von den ,produktivmachenden“ Kräften alkoholischer Getränke, was Spuren in seinen Trinkgedichten hinterlassen hat.

\section{3 ,Jiu酒“ oder „Wein“ als Motiv in den Trinkgedichten von Li Bai und Goethe}

Nach der durch die Kulturforschung bestätigten Einsicht der Gastrosophen kann man Trink- und Essensgenuss als Modus der sinnlichen und kommunikativen Selbstvergewisserung verstehen, und gemeinsames Trinken ist daher ein besonderes Medium der Verständigung, der Vertrauensbildung, des Gedankenaustauschs (vgl. Wierlacher 2003, 171). Wann und wo wird getrunken, warum wird getrunken, mit wem wird getrunken usw., diese Fragen berühren kulturelle Aspekte des Trinkens. Echte Freunde bedeuten eine angenehme Gesellschaft und können eine behagliche Atmosphäre erzeugen. Auch bei Li Bai und Goethe sind Trinken und Freundschaft eng miteinander verbunden. In Goethes Gedicht Tischlied sind die folgenden Verse zu lesen:

\footnotetext{
Freunden gilt das dritte Glas,

Zweien oder dreien,

Die mit uns am guten Tag,

Sich im Stillen freuen

Und der Nebel trübe Nacht,

Leis und leicht, zerstreuen,

Diesen sei ein Hoch gebracht,

Alten oder neuen.

(Von Goethe 2007a, 172-173)
}

Auch Li Bai trank gern in Begleitung von Freunden, so heißt es in seinem Gedicht Mit dem Bergeinsiedler zechend (山中与幽人对酌): 
Selbander sitzen wir beim Trunk;

两人对酌山花开, im Berge blüht der Hain.

Ein Becher und ein Becher und

一杯一杯复一杯。 noch einmal ein Becher Wein.

Ich bin berauscht und möchte schlafen.

我醉欲眠卿且去 Hochwürden, geht auch Ihr!

Seid morgen früh, wenn's Euch gefällt,

明朝有意抱琴来。 mit Eurer Zither hier. ${ }^{6}$

Mit seinem Freund hat das lyrische „Ich“ einen Becher nach dem anderen geleert und das Trinken sehr genossen, weil die Chemie zwischen den beiden stimmt. Das entspricht genau einem in China sehr bekannten Spruch 酒逢知已千杯少 [wörtlich: ,Trinkt man mit einem gleichgesinnten Freund, so sind tausend Gläser Schnaps zu wenig!‘]. Sogar im betrunkenen Zustand vergisst das „Ich“ nicht, ein neues Zusammensein - und das heißt hier auch: zusammen trinken - für den nächsten Morgen zu vereinbaren. Interessanterweise ging der lyrische Li Bai sogar so weit, dass er sich die Natur zum Trinkkumpan einlud, wenn einmal kein Freund zur Stelle war. So heißt es in seinem berühmten Gedicht Der einsame Zecher im Mondenschein (月下独酌) zu Beginn:

$\begin{array}{ll}\text { 花间一壸酒, } & \text { Nur Blüten rings und dieser Krug mit } \\ & \text { Wein, } \\ \text { 独酌无相亲。 } & \text { alleine trinke ich, kein Freund hält mit. } \\ \text { 举杯邀明月, } & \begin{array}{l}\text { Ich heb den Becher, lad den Mond mir } \\ \text { ein, }\end{array} \\ \text { 对影成三人。 } & \text { mit meinem Schatten wären wir zu dritt. }{ }^{7}\end{array}$

Diese Szene des Zechens birgt den klassischen chinesischen philosophischen Gedanken in sich, nämlich den Gedanken der „Einheit von Himmel und Mensch“ (天 人合一). Demnach stehen sich Mensch und Natur nicht gegenüber, wie es in den westlichen Philosophien oft der Fall ist, sondern die beiden Größen bilden eigentlich eine harmonische Einheit. In der oben genannten Strophe hat das lyrische „Ich“ seiner kühnen Phantasie freien Lauf gelassen und den Mond sowie seinen

6 Das chinesische Original stammt von Li Bai 1983, 264. Übersetzt von Günter Debon (vgl. Debon 2009, 61).

7 Das chinesische Original stammt von Li Bai 1983, 264. Übersetzt von Volker Klöpsch (vgl. Klöpsch 2009, 67). 
Schatten im Mondschein als zwei Zechkumpane betrachtet. Aber genau das hebt die Einsamkeit des „Ich“s noch mehr hervor.

Bei Goethe wird das Alleintrinken nicht mit der Einsamkeit des Alleinzechers in Verbindung gesetzt, sondern eher mit geistigem Schaffen, mit den ,produktivmachenden Kräften sehr bedeutender Art“, wie in seinem Gedicht Das Schenkenbuch geschildert:

\author{
Meinen Wein \\ Trink' ich allein; \\ Niemand setzt mir Schranken, \\ Ich hab' so meine eignen Gedanken. \\ (Von Goethe 2007a, 378)
}

Für einen Sorgenbrecher hielt Goethe den Wein. Seiner Meinung nach kann der Wein eine Trunkenheit seelisch-geistiger Natur erwecken, kann beflügeln, im Alter helfen, sich jung zu fühlen und dadurch die Lebenssorgen vorübergehend loszuwerden. Dies wird in der folgenden Strophe in Das Schenkenbuch zum Ausdruck gebracht:

\footnotetext{
Trunken müssen wir alle sein!

Jugend ist Trunkenheit ohne Wein;

Trinkt sich das Alter wieder zu Jugend,

So ist es wundervolle Tugend.

Für Sorgen sorgt das liebe Leben

Und Sorgenbrecher sind die Reben.

(Von Goethe 2007a, 379)
}

In dieser Strophe hat Goethe „Jugend“ metaphorisch als „Trunkenheit ohne Wein“ dargestellt, mit dem Unterton, dass der Wein rauschhafte Gemütszustände erzeugen kann, wie z.B. Begeisterung, Übermut, Glücksgefühl, Ekstase usw.

Bei Li Bai gilt das ,Jiu酒“ auch oft als Sorgenbrecher, was sich deutlich in den folgenden Versen im Gedicht Beim Abschiedsmahl auf dem Turm des Xie Tiao in Xuanzhou. Für meinen Onkel, den Sekretär Li Yun geschrieben (宣州谢朓楼饯别校 书叔云) zeigen lässt:

抽刀断水水更流，

举杯消愁愁更愁。

人生在世不称意,
Doch teile nur mit dem Schwerte das Wasser das Wasser bleibt weiter im Fluß.

Ertränke ruhig im Wein den Verdruß Verdruss kommt noch zum Verdruß.

Solange der Mensch auf Erden weilt, ist ihm die Erfüllung versagt - 


\title{
明朝散发弄扁舟。 \\ das Haar drum gelöst und im Kahne hinfort morgen, sobald es tagt! ${ }^{8}$
}

Zwar kann man das Jiu酒 als Sorgenbrecher einsetzen, wenn man im Leben mit Schwierigkeiten konfrontiert wird oder unter negativen Gefühlen leiden muss, aber Li Bai hat doch die bittere Erfahrung machen müssen, dass alle Sorgen ${ }^{9}$ immer noch Sorgen bleiben, sobald man aus dem Rausch erwacht, versinnbildlicht in einem Vergleich zwischen dem Wasser, das man mit einem Schwert zu teilen versucht, und dem Verdruss, den man im Alkohol zu ertränken versucht. Ungeachtet dieser Einsicht wendet man sich weiterhin an Jiu酒 als Sorgenbrecher. Weil man auf die vorübergehende Erlösung aus den Kümmernissen nicht verzichten mag?

In der westlichen Literatur wird das Thema „Wein und Liebe“ bis heute immer wieder aufgegriffen. So wird etwa im Hohelied Salomos, einer Sammlung von zärtlichen, teilweise erotischen Liebesliedern aus dem Alten Testament, die Geliebte mehrfach mit Wein in Beziehung gebracht. In diesem Zusammenhang war auch Goethe keine Ausnahme. Er sieht eine Verbindung zwischen Rose und Rebe, nämlich der Königin der Blumen und der Königin der Früchte. Diese Verbindung hat er in den folgenden Versen von Nachgefühl angedeutet:

\author{
Wenn die Reben wieder blühen, \\ Rühret sich der Wein im Fasse; \\ Wenn die Rosen wieder glühen, \\ Weiß ich nicht wie mir geschieht. \\ (Von Goethe 2007a, 105)
}

\footnotetext{
8 Das chinesische Original stammt von Li Bai 1983, 130. Übersetzt von Volker Klöpsch (vgl. Klöpsch 2009, 92).

9 Li Bais Sorgen gingen in erster Linie auf die Niederlage in seiner beruflichen Tätigkeit zurück. Im Jahr 742 wurde er auf Empfehlung von He Zhizhang (贺知章), einem berühmten Dichter - und zugleich kaiserlicher Sekretariatschef -, von Kaiser Ming-huang zum Beamten der renommierten Hanlin-Akademie ernannt. Zwar bewunderte der Kaiser sehr die Schaffenskraft des trinkfreudigen Dichters, aber dessen freimütige und ungebundene Art führte dazu, dass er den Eunuchen Gao Lishi (高力士) demütigte und nur zwei Jahre später aus dem Staatsdienst entlassen wurde, vermutlich aufgrund einer Intrige des Eunuchen. Danach ging er landesweit auf Wanderschaft. Inzwischen lernte er in Luoyang Du Fu kennen und begann mit ihm eine intensive und jahrelange Freundschaft. Die beiden machten sich damals schon Sorgen um die politische Lage des Landes. Seit Anfang 755 gehörte Li Bai zur Gruppe des rebellierenden Kaisersohns, des Prinzen Lin von Yung (永王李璘). Nach dem Scheitern von dessen Aufstand wurde Li Bai als Konspirator ins Gefängnis geworfen, begnadigt und am Ende nach Yelang (夜郎) verbannt (Vgl. Li Bai 1983, 2-5; Debon 2009, 8-9).
} 
Die letzte Zeile in dieser Strophe weist wohl darauf hin, dass der Sprecher Schmetterlinge im Bauch hat. Für Goethe gehören Liebe, Lied und Wein zusammen, sie wirken harmonisch aufeinander ein, wie in den Strophen von Elemente gezeigt wird:
Liebe sei vor allen Dingen
Unser Thema, wenn wir singen;
Kann sie gar das Lied durchdringen,
Wird's um desto besser klingen.
Dann muss Klang der Gläser tönen,
Und Rubin des Weins erglänzen:
Denn für Liebende, für Trinker
Winkt man mit den schönsten Kränzen.
(Von Goethe 2007a, 310)

Goethes Auffassung über die Wechselbeziehung zwischen Wein und Liebe kommt wohl in den folgenden Versen von Das Schenkenbuch am deutlichsten zum Ausdruck:

\author{
Denn meine Meinung ist \\ Nicht übertrieben: \\ Wenn man nicht trinken kann \\ Soll man nicht lieben; \\ Doch sollt ihr Trinker euch \\ Nicht besser dünken, \\ Wenn man nicht lieben kann \\ Soll man nicht trinken. \\ (Von Goethe 2007a, 379)
}

Im Vergleich zu Goethe hat Li Bai das Thema ,Jiu酒 und Liebe“ kaum explizit behandelt. Dieser Unterschied lässt sich wohl wie folgt erklären: Unter dem Einfluss des Dionysos-Geistes neigen die westlichen Dichter dazu, den rauschhaften Gemütszustand eines Menschen bis in die letzten Feinheiten darzustellen. Es ist kaum zu leugnen, dass ein Glas Wein stimmungsaufhellend wirkt, einen aufgeschlossen macht und dadurch die Kontaktfreudigkeit steigert, wie ein Zitat von Ovid - „Wein macht die Herzen bereit und öffnet sie den Liebesgluten“ - illustriert. Die chinesische Kultur aber ist vornehmlich durch den Konfuzianismus geprägt, unter seinem Einfluss steht auch die chinesische Dichtung. Im Buch Gespräche (论语) hat Konfuzius einmal seinen Schülern gegenüber den Nutzen vom Buch der Lieder (诗经), der frühesten Sammlung chinesischer Dichtung, so kommentiert:

《诗》可以兴, 可以观, 可以群, 可以怨; 迩之事父, 远之事君; 多识于 鸟兽草木之名。 
[,Die Lieder sind geeignet, um anzuregen; geeignet, um $\mathrm{zu}$ beobachten; geeignet, um zu vereinigen, geeignet, um den Groll zu wecken; in der Nähe dem Vater zu dienen, in der Ferne dem Fürsten zu dienen; man lernt (außerdem) viele Namen von Vögeln und Tieren, Kräutern und Bäumen kennen.' $]^{10}$

Damit hat Konfuzius den Grundton für die chinesische Dichtung festgelegt, und zwar eine politisch-didaktische Funktion. In einen solchen Zusammenhang passt weder eine Verherrlichung des Weines noch der Liebe. Zwar war Li Bais Dichten bekanntlich im Wesentlichen taoistisch inspiriert, aber unter der alles dominierenden konfuzianischen Gesinnung konnte sich keine Tradition ekstatischer Lyrik herausbilden. Als Inbegriff des Unkontrollierten, Anarchischen, Schamverletzenden spielt die erotische Liebe in der chinesischen Poesie keine solche Rolle wie in der abendländischen. So findet sich auch in der Dichtung von Li Bai kaum eine Verbindung Liebe - Wein.

Wie oben erwähnt, stellt das gemeinsame Trinken eine Form der Kommunikation dar. Dabei spielt in der chinesischen Kultur das Quanjiu (劝酒, wörtlich: ,jemanden zum Trinken überreden') eine besonders wichtige Rolle, die einem westlichen Beobachter ungewöhnlich vorkommen mag. Zumindest hinsichtlich der Quantität und der Intensität scheint dem Alkoholtrinken unter Freunden oder Geschäftsleuten in China mehr Wert beigemessen zu werden als in der deutschen Trinkkultur. Es gibt z.B. ein paar bekannte Sprüche, die man gerne benutzt, wenn man dem anderen einschenken oder nachschenken möchte, wie 大海航行靠舵手, 增进友谊靠喝酒 [,Was der Steuermann für die Seefahrt ist, ist das Alkoholtrinken für die Freundschaft']. Beim Trinken mit Freunden setzte der lyrische Li Bai auch gerne seine hohe Überredungskunst ein, indem er von seiner Lebensphilosophie bzw. Lebensweisheit sprach, z.B. im Gedicht Hier kommt der Wein (将进酒), das als eines der berühmtesten Trinklieder von Li Bai gilt und das das ungezügelte Temperament des Dichters am besten und am deutlichsten widerspiegelt:

$\begin{array}{lc}\text { 君不见黄河之水天上来, } & \begin{array}{c}\text { O siehst du nicht } \\ \text { Die Wasser des Gelben Stromes: } \\ \text { Vom hohen Himmel gekommen, }\end{array} \\ \text { 奔流到海不复回! } & \begin{array}{c}\text { Jagen sie meerwärts } \\ \text { und kehren nie mehr zurück! } \\ \text { O siehst du nicht }\end{array} \\ \text { 君不见高堂明镜悲白发, } & \begin{array}{c}\text { Droben im Saal den Spiegel } \\ \text { betrauern dein weißes Haar? }\end{array}\end{array}$

10 Das chinesische Original stammt von Yang 2010, 196. Übersetzt von Richard Wilhelm (vgl. Wilhelm 2015, 136). 
朝如青丝暮成雪!

人生得意须尽欢,

莫使金樽空对月。

天生我材必有用,

千金散尽还复来。
Morgens noch war es wie dunkle Seide; abends schon ward es zu Schnee.

So lebe, Mensch, nach Herzenslust und koste bis zur Neige das Glück!

Laß nie den goldenen Pokal leer unterm Monde stehn!

Der Himmel schuf in uns die Gaben so müssen wir sie nützen.

Wenn tausend Gulden verschleudert sind sie kehren wieder ein andermal. ${ }^{11}$

Aus den oben zitierten Strophen können wir die Lebensphilosophie ablesen, dass man das Leben rechtzeitig genießen sollte, weil die Zeit unwiederbringlich vergehe. Dazu diene das Trinken als ein hervorragendes Hilfsmittel. In diesem Kontext forderte Li Bai sein Gegenüber weiter zum Trinken auf:

\author{
烹羊宰牛且为乐， Kocht einen Hammel! Schlachtet ein Rind! \\ Und laßł uns fröhlich sein! \\ 会须一饮三百杯。 Laßt uns auf einen Zug \\ dreihundert Becher leeren! \\ 岑夫子, 丹丘生, \\ Du, Meister Tsen! Mein Freund Zinnoberbüh!! \\ 将进酒, 杯莫停。 \\ Hier kommt der Wein. Weis' keiner ihn zurück! $!^{12}$
}

\title{
4 Fazit
}

Als ein Nahrungsmittel haben Wein oder Jiu酒 nicht nur einen körperlich-materiellen Aspekt, sondern auch einen psychisch-soziokulturellen; daher ist das Trinken als Nahrungsaufnahme ein kulturell-psychisch-gesellschaftlich geprägter Akt und mehr und etwas anderes als eine Funktion des Stoffwechsels (vgl. Wierlacher 2003, 166, 168). Um das Trinken herum ist die Trinkkultur entstanden, die als kulturelles Kollektivgedächtnis einer Menschengruppe gespeichert wird und schon in ihrer Kultur integriert ist. Das Zusammenspiel dieser Art Kultur mit anderen

11 Das chinesische Original stammt von Li Bai 1983, 116. Übersetzt von Günter Debon (vgl. Debon 2009, 41).

12 Das chinesische Original stammt von Li Bai 1983, 116. Übersetzt von Günter Debon (vgl. Debon 2009, 41). 
Faktoren, beispielsweise dem Geschmack im physischen Sinne und der Atmosphäre einer konkreten Kommunikationssituation, wie z. B. wann, wie, warum, mit wem usw. man trinkt, bestimmt erst, wie Wein oder Jiu酒 einem schmecken und wie man den Gegenstand des Trinkens wahrnimmt. Verschiedene Gefühle und Assoziationen werden hervorgerufen, die einen zu einem Gedicht usw. inspirieren können, wie hier in den Fällen von Li Bai und Goethe. Eine komparative Analyse könnte u.a. zu dem Schluss kommen:

Der Gegenstand des Trinkens in den Trinkgedichten von Li Bai und Goethe ist oft nicht gleich. Die chinesische Traubenweinkultur kann zwar auf eine lange Geschichte zurückblicken, aber gegenüber anderen alkoholischen Getränken wie Reiswein oder Schnaps hat sie im Laufe der Zeit mehr oder weniger an Popularität verloren. Dies lässt sich meines Erachtens wohl auf zwei Gründe zurückführen: Erstens ist China aufgrund der geographischen Lage und der Klimabedingungen seit alter Zeit ein Agrarland und reich an verschiedenen Getreidesorten, während Weintrauben in China eigentlich ein Importgut und für längere Zeit exklusiv den Gelehrten und den höheren Schichten der Gesellschaft zugängig war; zweitens misst die Weinkultur dem Schmecken und Abschmecken beim Weintrinken große Bedeutung bei und fordert deswegen eine relativ langsamere Genussreise mit Hilfe von Nase und Zunge, was dem Alkoholkonsum im chinesischen Kontext meistens nicht entspricht. Dem chinesischen Trinker geht es weniger um die Erschließung der Qualitäten des Alkohols, weniger um den Genuss des Alkohols als darum, durch das Alkoholtrinken soziale Hierarchien zu demonstrieren und $\mathrm{zu}$ festigen oder auch ein Gefühl der Solidarität zu unterstützen; es geht also in erster Linie um die Durchführung einer Zeremonie oder eines Rituals. In diesem Zusammenhang bezieht sich das, was man trinkt, in Li Bais Trinkgedichten nicht häufig auf den Traubenwein. Aber es ist interessant $\mathrm{zu}$ beobachten, dass das Wort „Wein“ fast bei allen deutschen Übersetzungen von Gedichten Li Bais als die Entsprechung von „Jiu酒“ auftaucht. Es hängt wohl mit den Assoziationen des Wortes „Wein“ in der deutschen Kultur zusammen. „Wein“ hat eher eine poetische Aura und könnte oft angenehme Gefühle und liebenswürdige Assoziationen beflügeln, was am besten auf den poetischen Gestus des „Jiu酒“ in Li Bais Trinkliedern zutreffen würde. Mit einer solchen Übersetzungsstrategie könnte die Dichtung Li Bais besser dem Vorstellungsbild und den auf Ekstase zielenden Erwartungen des deutschen Publikums entsprechen.

Zwar werden Jiu酒 oder Wein sowohl von Li Bai als auch von Goethe als Sorgenbrecher betrachtet, aber der Wein scheint bei Goethe ein wirksames Mittel gegen die Sorgen des Lebens zu sein, während Li Bai sich eher über die Tatsache im Klaren ist, dass man den Verdruss eigentlich gar nicht ertränken kann. Durch dieses Bewusstsein der Hilflosigkeit gewinnt sein Verdruss noch an Intensität und ergreifender Kraft. 
Li Bai und Goethes Trinkgedichte spiegeln einflussreiche Elemente der chinesischen und der deutschen Kultur wider. In der chinesischen Kultur übt der philosophische Gedanke „Einheit von Himmel (Natur) und Mensch“ bis heute einen weitreichenden Einfluss aus. Daher ist es nachvollziehbar, weshalb sich Li Bai beim einsamen Zechen den Mond und den eigenen Schatten als Kumpane vorgestellt hat. Bei ihm handelt es sich nicht um eine Gegenüberstellung von Mensch als Subjekt und Natur als Objekt, sondern um die harmonische Co-Existenz und Interaktion zwischen den beiden. Außerdem findet auch die gesellschaftliche Konvention von Quanjiu Chinas ihren Niederschlag in Li Bais Trinkgedichten. Die deutsche Trinkkultur scheint eher vom Geist des Dionysos geprägt zu sein, und Goethes Trinkgedichte haben oft die Kombination von Trinken, Lebensfreude und Liebe als Thema. Diese Kombination bei Goethe lässt sich auch auf die anakreontische Dichtung aus der Mitte des 18. Jahrhunderts zurückführen, zu deren bevorzugtem Themenkreis Liebe, Freundschaft, Geselligkeit und Weingenuss gehören und die den jungen Goethe eben nicht wenig beeinflusst hat. Im Gegensatz dazu taucht dieses Thema nur selten explizit in der chinesischen Dichtung auf, da diese unter dem konfuzianischen Einfluss großen Wert auf die politisch-didaktische Funktion der Dichtung gegenüber dem Volk bzw. der Gesellschaft legt und allen „Ausflügen“ eines Dichters ins Anarchische, Ekstatische, Unkontrollierte, wenn nicht ablehnend, so doch reserviert gegenübersteht.

\section{Literaturverzeichnis}

Boehncke, Heiner, und Joachim Seng (Hg.). Goethe und der Wein. Berlin: Insel, 2014.

Christoffel, Karl. Der Wein in Goethes Leben und Dichtung. Bernkastel-Kues: Weinberg-Keller, 1978.

Debon, Günter. Li Tai-bo Gedichte: eine Auswahl. Stuttgart: Reclam, 2009.

Du, Fu. Du Fu Shixuanzhu [Du Fu Gedichte: eine Auswahl mit Kommentaren]. Ausgewählt u. kommentiert von Xiao, Difei. Shanghai: Shanghai guji chubanshe [Shanghaier Verlag für alte Schriften], 1983.

Guo, Canjin. „,Li Bai dou jiu zhi liang“ de shuju huansuan [Die Umrechnung des Jiu-Konsums in Kufe bei Li Bai]“. Guoxue [Sinologie in China] 8 (2012): 57.

Li, Bai. Li Bai Shixuan [Li Bai Gedichte: eine Auswahl]. Ausgewählt u. kommentiert von Fudan Daxue Gudian Wenxue Jiaoyanzu [Die Arbeitsgruppe für Lehre und Forschung der klassischen Literatur an der Universität Fudan]. Beijing: Renmin wenxue chubanshe [Verlag der Volksliteratur], 1983.

Klöpsch, Volker. Der seidene Fächer: Klassische Gedichte aus China. München: dtv, 2009. Kupfer, Peter. „Neue Weinkultur und der Wandel der Gastlichkeit in China“. Gastlichkeit: Rahmenthema der Kulinaristik. Hg. Alois Wierlacher. Berlin: LIT, 2011, 375-388.

Von Goethe, Johann Wolfgang. Goethe Werke in sechs Bänden: Erster Band. Hg. Friedmar Apel, Hendrik Birus, Anne Bohnenkamp, Dieter Borchmeyer, Hans-Georg Dewitz und Karl Eibl et al. Frankfurt/M. und Leipzig: Insel, 2007a. 
Von Goethe, Johann Wolfgang. Goethe Werke in sechs Bänden: Fünfter Band. Hg. Friedmar Apel, Hendrik Birus, Anne Bohnenkamp, Dieter Borchmeyer, Hans-Georg Dewitz und Karl Eibl et al. Frankfurt/M. und Leipzig: Insel, 2007b.

Von Zach, Erwin Ritter. Li T'ai-po. Gesammelte Gedichte: Teil 3. Wiesbaden: Harrassowitz, 2007. Wang, Shizuo, und Ping Huang. „Lun Zhongguo de putaojiu wenhua [Über die Traubenweinkultur in China]“. Niangjiu keji [Technik des Jiu-Herstellens] 11 (2009): 136-143.

Wierlacher, Alois. „Kultur und Geschmack“. Handbuch interkulturelle Germanistik. Hg. Alois Wierlacher und Andrea Bogner. Stuttgart und Weimar: J. B. Metzler, 2003, 165-175. Wilhelm, Richard. Konfuzius Gespräche. Hamburg: Nikol, 2015.

$\mathrm{Xu}$, Shen. Shuowenjiezi [Erklärung einfacher und Analyse zusammengesetzter Schriftzeichen]. Beijing: Zhonghua Shuju [China Verlag], 2017.

Yang, Bojun. Lunyu Yizhu [Erläuterte Übersetzung der Gespräche]. Beijing: Zhonghua Shuju [China Verlag], 1980.

Yang Zhang, geb. 1983; Promotion 2013 an der Fremdsprachenuniversität Beijing; seit 2014 Dozentin an der Deutschabteilung der Südwest Jiaotong Universität; seit 2017 Leiterin des vom NSSFC geförderten Projekts zum Thema „Übertragung und Erforschung der Tang-Dichtung im deutschsprachigen Raum“. 
